\title{
"Montage" Mentality on the Art of Painting —Study about Figure Painting Scenes of Convergence in the Five Dynasties and Song Dynasty
}

\author{
Zhihui Wang \\ Qinhuangdao Institute of Technology, Qinhuangdao, Hebei, China
}

\begin{abstract}
We often see different time and space scenes, different stories and different viewpoints change organically through one lens converge to form a complete film in the movie, and one of a series of the shearing lens elements and convergence is the montage technique. Such a method actually also often exists in different scenes in our ancient literature and different scenes and jumping thinking thoughts form a series of montage in the mind, and then it can be expressed through poetry. This case is the same as it in art, different time and spaces, the characters appear in a scene in a picture, and we distinguish them with a series of symbols to convergence in each scene. Therefore, we can say that if the montage was born after his birth of firms in the twentieth century, then it has existed since ancient time, and it has always existed in the fine arts, however, such thinking played an important role in the development of Chinese art, especially in the convergence and performance practices screen scene.
\end{abstract}

Keywords-montage thinking; thinking: song figures; convergence of scenes

\section{MONTAGE THINKING AND SCENES CONVERGENCE IN THE PICTURE}

\section{A. What is a montage of thinking?}

In daily life, when we are observing, understanding, perfuming surrounding things,"either through continuous tracking without destroying the unity of the real time and space, of course, it can disrupt the unity of the real time and space to carry out, attached see (or want to), and leaping to see (or think) are two basic methods for the people to observe, recognize and thus they are the performance of real life. "Continuation and jumping, completion and segmentation, stationary and motion are the basic manifestations about everyday people thinking, psychological, emotional possess. When faced with a series of individual factors, we will take these factors into a unified, complete image in mind through the clip, polymerization and other methods, and this " montage thinking is that individual will be aggregated into the whole through segmentation, the dispersed, jumping way of thinking, the whole has entered the rules and methods in people's consciousness and emotions through the image.”

In the stations of the ancients rare cases, a section of the event describe it with a concise language, and then they connect it into a complete narrative in order to express ideas. "Broken Bamboo", "Flying soil"," the dead", "abandoning" like a movie scenes and lens. Whether modern people or, the ancients thousands of years ago, they can easily appreciate the meaning conveyed. Of course, if such literary scene was transformed into painting, then as "broken bamboo" "Flying earth" and so on, they like a piece of the screen scene, several scenes become a complete synthesis of painting scroll--- such as "Han Banquet map ", five scenes are nature, specific and detailed record to describe all the details of the banquet, rather than liking ancient as only one or two simple phrases to describe. Painting language and rich expression make the work more complete, but thinking of montage is still the same strain, and it does not degenerate with the passage of the times. Based on this, I think, it is really product after the 19th century montage cinema, but montage thinking always exists from the ancient time, and it has always existed in the painting. Such assumptions help us explore the convergence and performance practices of Chinese painting in the scene from a new angle, and then to understand intrinsic heritage and development history of the art painting.

\section{B. Montage thinking in the art}

For more narrative montage of thinking, it could easily cause a misunderstanding that the role of the individual is greater than the whole. Each scene or picture is an individual, and the individual fragments creators make individual select, crossover recombine, thus it can make the overall link, the result is the process of "local - global" group. In this perspective, it is right, but if there are a whole thinking and architecture, it only has every piece scenes, clips, lens, and then the final result can be imagined. Because of this, we should establish a "global - local - the whole" concept in the creative process, and this is what the full montage thinking is. Eisenstein, former Soviet Union, the famous director, Eisenstein's montage theorists, believes that life is same with art, everywhere there is a montage thinking, it is "a series of appearances by this or that way, it gradually formed the complete image though the various factors in people's feelings in mind. "When we want to remember the People's Square in mind, we naturally think that the People's Square is a holistic Square and space, and it includes the overall concept liking the Grand Theatre, Shanghai Museum, the local details, Urban Planning Exhibition Hall, the municipal government. But when we are being in the People's Square, we always think we have seen all buildings. In fact, we do not see everything, we might just see a specific scene Shanghai Grand Theatre, Museum only, that is to say, I just see some of the most important scenes due to the continuous changes of attention to it, and these fragments scene constitute the overall impression of the whole People's Square together. 
Similarly, "we all thought that we're aware of what one sees all the time, in fact we only are aware of some major moments, and we put these things in a short period of time consisting of elapsed time by the continuity of awareness activities." The montage thinking that leaves out the middle part exists everywhere in life, and it is in appreciation of the arts and creation too. Ancient Chinese painter minds theory "to intended first before pen", decided general concept which has been on the minds of the images before the painting, and then they select the scene by subjective, partial fragments to make different combinations of elements as a whole to form a complete picture; but when the viewer enjoy this kind of work, such as works "Han Banquet map", it does not produce incoherent or incomprehensible difficulties.

Therefore, similar montage thinking is similar with Gestalt psychology Gestalt structure. Gestalt psychology thinks that when we enjoy a whole or we are in the creation of drawings, "what the various elements look like depends on the place of factor in the location and the effect they make. When eyes are observing the simple act of thinking in such a simple line drawing pattern, it always appears the pursuit of unity and a positive tendency about the sense of order. "Because people have such Gestalt psychology thinking, so when there are gaps in one of a triangle, we do not believe that this is not a triangle graphics. It is the same with enjoying the paintings, contents do not lack continuity because of the performance of a scene and another scene, and then it won't deny that it is a continuous story. It is this holistic thinking, which makes us not be confused for the scene under temporal convergence of different spatial when we are in the creation or appreciation, and it is a major cause that montage gets improved in the evolving art.

\section{Montage thinking and symbolic link in scenes picture}

Each one has a number of scenes to be composed, and the scenes of the picture like montage paragraphs, if those paragraphs use a professional movie terminology to explain, that is the "basic chain", each "basic chain" containing a montage thinking forms a complete work through segmentation. Just as literature is formed by dividing through punctuation and it makes easy paragraph or sentence reading for our reading, movies make combination and the length of the lens switching to change movie screen, the convergence symbol of scenes is also indispensable and ubiquitous in painting. The folding screen of Gu Hongzhong's "Han Banquet map" are taking two or more scenes into a symbolic picture of convergence, these items not only play a role in dividing the scene or to give the audience effects about convergence before and after the scene causing, but also they play role in the whole construction works. In other words, these symbols are not a simple tool, but they have the actual content and significance. But the dual function of this symbol does not existed in art at the beginning, and it also is the result of a series of changes before they split from a single object to become a part of the picture.
Art in the development process, paintings with montage thinking requires a relatively large space, or a carrier. If you do not have a relatively large space, then the different clips in the story could not link together organically. Long scroll that Chinese figure painting particularly has is the best creative thinking carrier of montage, so if there is no scroll painting carrier, we will not see five or six scenes appear in the same picture through space and time. This article examines the development of the Northern Song Dynasty Qin Dynasty to Chinese figure painting from the perspective of montage thinking.

\section{SONG PORTRAITS OF "MONTAGE OF THINKING" AND THE SCENE OF CONVERGENCE SKILLS}

Song Dynasty is the pinnacle of Chinese figure painting, and "Along the River during the Qing Ming Festival" undoubtedly stands in the apex of the pearl. The volume uses realistic expression and panoramic composition skills to describe social life in Kaifeng coast near the river, the Northern Song Dynasty capital, outside the East Gate and the rich social style. From visual narrative units setting and visual program of environmental setting, the whole picture can be broadly divided into "suburban landscape", "near the river traffic", "downtown Kaifeng". Three visual paragraphs are composed by two aquatic and terrestrial environments. On the five-meter-long scroll, it arranges different scheduling scenarios through the "water" and "land" to lay the screen images, which is similar to the movies using the organization "water" and "land" to schedule screens, and it shows the life form the suburbs to the downtown scene. Business structure out of 'water' and 'land' environmental systems composed of two cross composite visual programming that " Riverside Scene at Qing Ming Festival" has is very close to the intersection of film narrative montage program narrative structure. They both use an independent narrative way to explain the activities of the two space alone, but also they use the narrative structure about the cross-montage to organize space, and they use the means of zoom lens to explain the depth relationship of two spaces, so the viewers have feeling that they deep into a scene, and it can clearly identify images with a certain spatial depth of laying." Whole picture makes alternate interludes in two closely related narrative thread" between water "and" land ", it has caused composition density, and scene did not use mark delimiters do converge in the past, but it will not cause confusion distinction, we can see that the performance of the technique is very superb between each segment. Also paying attention to dramatic changes in rhythm and the rhythm of the story enhanced the sense of unity and complex compact narrative structure theme.

Climax part of the screen is huge transport ship through Hongqiao tense scenes. The climax is the artist skillfully "water" and "land" junction of two superimposed narrative environment, that "rainbow" is equivalent to the scene convergence symbols of entire picture, it is the intersection point that separated from each other and connects the two spaces, and it is the visual center of the segment, the convergence of these scenes role in the past will still be 
hidden to appear here in the picture, which suggests that the artist is still not completely out of the tradition. At this juncture, the artist describes a noisy marketplace above the bridge, busy bustling scene under the bridge. Stalls connected to each other in the beginning of the bridge, people on the left side of the parapet leisurely enjoy the scenery, and people on the right watched the boats countercurrent bridge railings. Deck Central describes that dignitaries met with each other and their servant slaves wanted people to give way, the screen quietly became congestion tension. Then, the screen are from the people nervously watching a transport ship to another tense scene: a large transport ship was passing the bridge head, the boat was still under the bridge, the end part of boat oars was exposing on the right side of the ship. Another cargo ship was about to walk through the bridge, the boat was horizontal, and it has been washed down the rapids. Boatmen were in disorder, some were struggling to punt, and some were waving and calling, tensions and rush appeared thoroughly. "Boat" and "bridge", the two intersecting narrative scenes become the hectic and stressful center, making the screen showing a strong dramatic plot conflict, it is amazing and memorable. "In the microscopic structure of the two narrative picture processing unit cross narrative space environment, the artist used the great changes in artistic to cleverly express that scene is big and small, far and near, sparse, dense, dynamic and static, simple and so on of the complex relationships, which is very similar to micro-handling skills in a variety of lens montage choosing to use in the film, "the famous director Zheng Junli has a wonderful statement:." The painter makes the colorful scenes of life in this narrow focus picture, sometimes he puts the characters on the status of vision, and he uses depth and complexity level to describe the atmosphere of the vast space, sometimes he puts the characters in close-range position to highlight a group of people making communicate with each other and their smiles, sometimes the scene is in the foreground (such as trees, stern, towers), which results in a similar close-up feeling. Painters make kinds of scenes into one continuous stream from moving point of view, when we move sight along the line of scroll, and we see the vision similar with the film's visual sliding lens. "

\section{SUMMARY}

If an individual is removed from history to analyze, it has no special significance, but this painting is only placed in a larger development framework in order to make them have a clear meaning, and once the target of a large style or theme becomes interesting object of study and confirms, then the analysis which is similar to the structuralism can be used to reconstruct the form of the subject and it can provide answers to each other's style of their evolution. More scenes with montage thinking during the different periods of Western and Eastern are put in a picture under a large structure to study, we can very well understand the different content about the convergence screen symbols, expression, scene content and their respective under different scenarios, and it provides a new perspective and ideas for the study of art history.

\section{REFERENCES}

[1] Soon Min existence in montage paintings [J] Yunyang Teachers College, 2013, 06: 105-108.

[2] Xu Yunchun Study of scenes convergence portraits in Song Dynasty and the Five Dynasties [J] folk art, 2014, 01: 150-153.

[3] Xu Yunchun "Montage" mentality in painting -the evolution of convergence in the scene of Chinese figure painting [J] aesthetic JOURNAL, 2014, 04: 95-105.

[4] Xu Yun Chun Painting Art "montage" thinking [D]. East China Normal University 2011.

[5] Li Yusheng Shallow theory of animation art and aesthetics of ink painting [J] Movie Review, 2012, 15: 34-36. 